\title{
An artist's inquiry into scoliosis: an adult case report Laura Ferguson
}

\author{
Address: New York, NY, USA \\ Email: Laura Ferguson - laura@lauraferguson.net
}

\author{
from 4th International Conference on Conservative Management of Spinal Deformities \\ Boston, MA, USA. 13-16 May 2007 \\ Published: 12 October 2007 \\ Scoliosis 2007, 2(Suppl I):P4 doi:I0.I|86/|748-7|6|-2-SI-P4
}

This abstract is available from: http://www.scoliosisjournal.com/content/2/SI/P4

(c) 2007 Ferguson; licensee BioMed Central Ltd.

\section{Objective}

To document improved symptoms in a patient who investigated the three-dimensionaldynamics of her own spinal curvature through visual imagery.

\section{Study design}

Idiopathic scoliosis was diagnosed at age eight years. A T5-T12 spinal fusion with cadaver bone graft, without instrumentation, was performed at age thirteen years (John Cobb, Hospital for Special Surgery, New York, NY, U.S.A). The Cobb angle of the post surgical curvemeasured eight-five degrees. By age thirty-two years restrictive lung disease, arthritis andspinal stenosis, were diagnosed. The symptoms worsened progressively.

\section{Methods}

An exercise program incorporating Alexander Technique, Yoga, Pilates, and neuromuscular training wasdesigned to (1) strengthen andstabilize muscles around hypermobile joints, and (2) improve proprioceptive awareness to allow postural adjustments to improve spinal alignment and symmetry. In collaboration with orthopedists and radiologists, images including a three-dimensional spiral computed tomography scan of the subject's skeleton were made for the purpose of drawing.

\section{Results}

Symptom stabilization occurred as the patient gained a more graceful and coherentsense of her body, as well as improved look and function. A series of artworks revealing the three-dimensionalspatial dynamic of the scoliotic spine were produced http://www.lauraferguson.net/.

\section{Conclusion}

Health related quality of life can be improved using movement practices based on these concepts. 